\title{
Lyapunov exponents for classical-quantum mixed-mode dynamics
}

\author{
Ersin Yurtsever \\ Department of Chemistry, Koç University, Istinye, Istanbul, Turkey
}

(Received 6 February 1996)

\begin{abstract}
The mixed-mode philosophy of combining classical and quantum degrees of freedom under a single umbrella is employed to study chaotic behavior under quantization. The quantal wave packet is expanded in terms of a set of basis functions. The Jacobi-Hamilton formalism of the time-dependent Schrödinger equation allows the treatment of real and imaginary components of the time-dependent expansion coefficients as coordinates and momenta so that Lyapunov exponents can be calculated. Under the mixed-mode formalism, a twodimensional nonlinearly coupled oscillator system is partially quantized by letting one of the modes obey classical and the other quantal dynamics. The Lyapunov exponent spectrum of the complete system is obtained and the results are compared with the fully classical ones. [S1063-651X(96)10510-9]

PACS number(s): 05.45.+b, 03.65.Sq, 82.20.Rp
\end{abstract}

\section{INTRODUCTION}

The search for the quantum signatures of classical chaos has attracted a great deal of attention [1-4]. As soon as it was established that nonlinear systems in general had rather uncommon but characteristic behavior of displaying highly unpredictable evolution in time, the questioning of the existence of similar dynamics for molecular systems had begun. Since the equations defining the motion are highly nonlinear in nature, one should expect to observe the signatures of chaos. Classical approaches to various vibrational problems indicated that even for very low-dimensional problems, one encounters the indications of chaos with irregular trajectories and large Lyapunov exponents. However, in all these problems, the interesting (and correct) physics can be described only in terms of the quantum mechanical methodology, and here the controversy arises. Does classical chaos manifest itself in the quantum world? Does the dynamics of a very small molecule display significantly different behavior upon changing some parameters such as energy or the magnitude of the external field? Obviously the characterization of the quantum dynamics and the relations to the corresponding classical dynamics is an interesting and open question.

Classically chaos is identified by the strong dependence on initial conditions which can be recognized in the study of the maximum Lyapunov exponent or the Kolmogorov entropy. These measures define the exponential separation of initially very close trajectories. A positive Lyapunov exponent implies that close lying points in the phase space may evolve quite differently in time, thereby pointing out that they forget their history rather quickly. Once the differential equations defining the time evolution are known, it is rather a simple task to compute these quantities, or if some final observations are known as time series, again there exist several algorithms to extract such information from them $[5,6]$. These calculations can be carried out routinely for problems of a few dimensions. But they become quite cumbersome when the dimensionality is increased, especially when all the exponents are to be computed. The analysis of Lyapunov exponents as functions of the perturbation parameters will point to the qualitative changes in the dynamics and can pinpoint the critical region where the regular dynamics ends and chaos begins. For few-dimensional systems, it is even possible to follow trajectories in various surfaces of sections of the phase space so that a visual inspection can be carried out for recognizing chaotic or regular motion. However, all of these measures are based on classical mechanics and do not apply directly in quantum mechanics. The wave mechanics states that the position and the momentum cannot be determined exactly due to the uncertainty principle. The average values over wave functions take the place of precisely determined observables and then the terms "phase space", and "phase space trajectory" lose their meanings. Consequently, defining "close lying" points in a similar manner becomes a rather difficult task.

Dealing with a discrete energy spectrum, one cannot define the "exponential separation," and other measures distinguishing the regular and "irregular' quantum dynamics must be found. If this distinction exists, then as $h$ goes to zero (classical limit), the "motion", should be chaotic or regular. There are two major components of the proposed criteria of identifying "irregularity" in quantum systems and the majority of these attempts are based not on dynamical but rather on static properties of the solutions of the Schrödinger equation. In general, eigenfunctions provide information on the localization and nodal structure of the solution [7-10]. If the classical system is integrable, the quantum Hamiltonian can be brought into separable form and its eigenfunctions are localized along certain modes (not necessarily along the original modes used in the definition of the Hamiltonian). So an analysis of eigenfunctions (usually in coordinate space) provides information on the localization and hence the separability of the Hamiltonian. Such eigenfunctions should also have well defined nodal structures of the separable operators. Alternatively one can study the decay of spatial correlation functions of wave functions [11] or follow time evolutions not in the classical phase space but in a similar phase space defined in expectation values of coordinates and momenta. Relatively fast decay of these space autocorrelation functions implies a degree of initial condition dependence. However, these measures rely on visual inspection and they are difficult to quantify, moreover the visualization tends to be problematic for more than twodimensional systems. In contrast, the statistical analysis of 
eigenvalues provides hard numbers as to the irregularity of the spectra [12-15]. The most common technique of analysis uses the distribution of the normalized nearest-neighbor spacings. If an $N$-dimensional Hamiltonian is separable, then the eigenvalue spectrum is in fact a superimposition of eigenvalues of $N$ one-dimensional Hamiltonians. Since these spectra are uncorrelated, the nearest-neighbor histograms should display a random (Poisson type) behavior. On the contrary, if the Hamiltonian is not separable, the eigenvalues are strongly correlated and the similar histograms show a Wigner-type distribution. There are two different functional forms whose limiting forms give Poisson and Wigner types of distributions which are driven by a single parameter $[12,16]$. The magnitude of this parameter defines the degree of irregularity so a transition from "regular" to "irregular" regions can be detected. Similar ideas based on this philosophy use the sensitivity of eigenvalues to perturbation parameters [17], distribution of intensities between adjacent states [18], and avoided crossings of eigenvalues upon varying perturbation parameters [19]. The complications arise in all these methods since a large number of highly accurate eigenvalues are required for a careful statistical analysis. In fact, we have even showed that statistics from inaccurate calculations may give qualitatively incorrect answers [20].

As all these methods for analyzing quantum dynamical results fail to produce definite evidence of chaos, in this work we resort to an approximate philosophy such that the effects of the quantization can be studied using classical mechanical tools. By defining "Lyapunov exponents" for a wave packet in a manner similar to the classical case, we look for the fingerprint of chaos in propagation of wave packets under the effect of a classical field. The idea of defining quantal Lyapunov exponents is not very common but there exist several attempts. The quantal distribution function in the so-called $Q$ representation has been proposed and has been shown to behave like a Lyapunov exponent [21] within a finite time regime. In a more recent report, the HamiltonJacobi formulation of the quantum mechanics is employed [22]. Here the gradient of the phase of the wave function and the quantum field are treated similarly to the classical momentum and the coordinate and their time evolutions are obtained from the Newtonian equations of motion for a onedimensional kicked oscillator.

Our approach is similar to that of Schwengelbeck and Faisal [22] in that it is based on the Hamilton-Jacobi formalism of the Schrödinger equation. However, instead of defining a quantum particle in a coordinate-momentum phase space, we proceed to expand the wave packet in terms of a basis set and label each basis function as a different particle which moves in the field of other "particles." In this case the dimensionality of the problem increases, however, the maximum Lyapunov exponent should still carry the characteristics of the problem.

Since our original interest in the problem of quantum chaos has its roots in the intramolecular vibrational relaxation [23], we study a two-dimensional nonlinearly coupled bound oscillator system. This model system can be used to describe the stretching vibrations of a three-atomic molecule and shows a strong classical chaos. Previously employing this model, again in a quantal-classical mixed-mode philosophy [24], we have studied how a classical particle behaved under partial quantization and have shown that the chaotic behavior of the classical particle was smoothed out due to the presence of the quantum field. Here we look at the other side of the problem, namely, what the dynamics of a wave packet looks like under the effect of a classical particle.

\section{MIXED-MODE APPROACH}

One of the standard approaches to the classical quantumcorrespondence is to apply both methodologies in a mixedmode (MM) fashion, that is, the part of the system is to be treated quantum mechanically where the remaining parts follow the rules of classical mechanics. Nowadays the classical dynamics of even very large systems of $10^{6}$ particles can be studied easily, whereas the quantum dynamics of more than three particles poses severe difficulties. Therefore one uses the mixed-mode philosophy to separate the problem into regions and solve the physically simple or uninteresting parts by classical methods. Once these regions are properly coupled to quantum degrees of freedom, the problem can be analyzed either by some approximate methods or using iterative techniques. In this manner very large scale problems can be brought into computationally manageable forms.

We have previously used this approach with a completely different aim. Let us define an $n$-dimensional Hamiltonian,

$$
H=\sum_{j} H_{j}+\sum_{j k} V_{j k}+\sum_{j k l} V_{j k l}+\cdots,
$$

where $V$ denotes terms which couple two or more sets of modes. If this Hamiltonian is not an integrable one due to the nonlinear character of $V$, the quantum dynamics can only be realized by solving the full-dimensional Schrödinger equation,

$$
i \hbar \partial \Psi(t) / \partial t=H \Psi(t) .
$$

The computational effort for the solution of Eq. (2) will have an $N^{n}$ dependence both for numerical and basis expansion methods where $N$ is the number of grid points or basis functions and $n$ is the dimensionality of the problem. Since this problem is intractable in the full quantum treatment especially for high $n$, a self-consistent-field (SCF) approximation can be applied in which the Hamiltonian is written as a sum of $n$ terms which include the couplings as averages [25-27],

$$
H=\sum_{j} H_{j}^{\mathrm{SCF}},
$$

with

$$
H_{j}^{\mathrm{SCF}}=H_{j}+\left\langle\sum_{k} V_{j k}+\sum_{j k l} V_{j k l}+\cdots\right\rangle_{n-1},
$$

with \langle\rangle$_{n-1}$ denoting an average over all modes except $j$. Then $n$ uncoupled (for a short time step) Schrödinger equations can be solved in an iterative manner. At each time step averages over all modes are computed, SCF Hamiltonians for that time step are set up, and the propagation of wave packets is computed under the average fields of remaining modes. Of course, being an approximate method, SCF carries certain errors and one has to analyze the magnitude of 
these errors. We have applied this method to several problems and compared the results with the exact quantum dynamics $[24,28,29]$. It seems that the quantum SCF approximation slows down the energy transfer between different modes. In fact, it has been suggested that to understand the time scales of energy transfer quantitatively, one has to resort to multiconfigurational SCF methods to overcome these errors [30].

However, in the mixing of classical and quantum dynamics, SCF seems to be a very convenient approach [31,32]. The averages over the classical modes used in the quantum Hamiltonians are not averages in the standard sense. Instead they are simple functions of classical observables (mostly the coordinates). Therefore, the only approximation in the quantum region is that the perturbation term remains constant for the duration of the integration step. As long as the time step is small enough, the errors due to this assumption must be very small. In fact we have shown that such an iterative process in a fully classical system did not change the qualitative description of the system [33]. On the other hand, the classical modes communicate with the quantum modes through so-called Ehrenfest couplings, which are averages over wavepackets. The justification lies in the Ehrenfest relations [34]:

$$
\begin{gathered}
d / d t\left\langle q_{j}\right\rangle=\left\langle\partial H / \partial p_{j}\right\rangle, \\
d / d t\left\langle p_{j}\right\rangle=-\left\langle\partial H / \partial q_{j}\right\rangle .
\end{gathered}
$$

The errors associated with this approximation depend on the separability of classical and quantum modes.

\section{HAMILTON-JACOBI FORMALISM FOR QUANTUM DYNAMICS}

The propagation of a wave packet can be obtained from the solution of the Eq. 2 [35,36]. For a one-dimensional problem, we proceed to expand the initial wave packet as a linear combination of basis functions (conveniently chosen as eigenfunctions of the uncoupled Hamiltonian),

$$
\Psi(q, t)=\sum_{\lambda} c_{\lambda}(t) \varphi_{\lambda}(q)
$$

where time-dependent coefficients can be written in terms of their real and imaginary components,

$$
c_{\lambda}(t)=x_{\lambda}(t)+i p_{\lambda}(t)
$$

Replacing the wave packet in Eq. (2) by Eq. (6), we obtain (in atomic units so that $\hbar=1$ and omitting the explicit time dependence of coefficients)

$$
i \sum_{\lambda}\left[\partial x_{\lambda} / \partial t+i\left(\partial p_{\lambda} / \partial t\right)\right] \varphi_{\lambda}=\sum_{\lambda}\left(x_{\lambda}+i p_{\lambda}\right) H \varphi_{\lambda}
$$

By multiplying with $\varphi_{\nu}$ from left and integrating over coordinates we obtain

$$
i\left[\partial x_{\nu} / \partial t+i\left(\partial p_{\nu} / \partial t\right)\right]=\sum_{\lambda}\left(x_{\lambda}+i p_{\lambda}\right)\left\langle\varphi_{\nu} H \varphi_{\lambda}\right\rangle
$$

In the most general case, Hamiltonian matrix elements can be complex:

$$
\left\langle\varphi_{\nu} H \varphi_{\lambda}\right\rangle=a_{\nu \lambda}+i b_{\nu \lambda} .
$$

Then equating real and imaginary components on both sides of the equation we get

$$
\begin{gathered}
\frac{\partial x_{\nu}}{\partial t}=\sum_{\lambda}\left(x_{\lambda} b_{\nu \lambda}+p_{\lambda} a_{\nu \lambda}\right), \\
\frac{\partial p_{\nu}}{\partial t}=-\sum_{\lambda}\left(x_{\lambda} a_{\nu \lambda}-p_{\lambda} b_{\nu \lambda}\right) .
\end{gathered}
$$

Equations (11) define the time evolution of basis coefficients. Let us define a formal Hamiltonian,

$$
H=(1 / 2) \sum_{\nu \lambda}\left(x_{\lambda} x_{\nu}+p_{\lambda} p_{\nu}\right)\left\langle\varphi_{\nu} H \varphi_{\lambda}\right\rangle .
$$

Then we note that

$$
\partial x_{\nu} / \partial t=\partial H / \partial p_{\nu}
$$

and

$$
\partial p_{\nu} / \partial t=-\partial H / \partial x_{\nu}
$$

which are the classical Hamilton-Jacobi equations of motion. Now the evolution of the quantum wave packet can be obtained employing well known methods of molecular dynamics simulations. In fact we have also shown that Newton formalism can be easily used at least for the timeindependent Hamiltonians [36].

Now the one-dimensional quantum problem (in coordinate representation) is transformed into an $2 n$-dimensional "classical-like" form and all the measures of classical nonlinear analysis can be applied.

\section{LYAPUNOV EXPONENTS}

Once the time-dependent Schrödinger equation is brought into the form of coupled nonlinear differential equations, we can define a new "phase space" in $2 n$ dimensions, where each dimension corresponds to a real or an imaginary component of a basis coefficient. We can then follow a phasespace trajectory, compute Lyapunov exponents, and observe whether the signs of classical chaos survive quantization. To compute Lyapunov exponents we use the tangent-space method, in which we look for the exponential divergence of $2 n$ points, which are infinitesimally close to the original point [37]. The regular updating of these points is a difficult problem and to work in the tangent space provides a reasonable formalism for the solution. Since the distances between the classical particle and $2 n$ points are very small, the time evolution of the distances can be brought into linear form,

$$
d / d t \delta x_{j}(t)=\partial F(\underline{x}) /\left.\partial \underline{x}\right|_{x=x(t)}, \quad \delta x_{j}(t),
$$

where $\delta x_{j}(t)$ is the distance of the point $j$ to the origin at time $t . x$ denotes all coordinates (and including momenta) and $F$ represents vector function for the time evolution of the point $x$. In our case $F$ is defined by Hamilton-Jacobi equa- 
tions [Eq. (11)] and $\partial F(\underline{x}) /\left.\partial \underline{x}\right|_{x=x(t)}$ is the Jacobian at given time $t$. Utilizing this algorithm with periodical orthonormalization of the vectors $\delta x_{j}$, we proceed to compute all Lyapunov exponents from the logarithms of the ratios of distances to initial separations.

The explicit forms of the $F$ and its Jacobian are given as

$$
\begin{gathered}
F\left(x_{\nu}\right)=\sum_{\lambda}\left(x_{\lambda} b_{\nu \lambda}+p_{\lambda} a_{\nu \lambda}\right), \\
F\left(p_{\nu}\right)=-\sum_{\lambda}\left(x_{\lambda} a_{\nu \lambda}-p_{\lambda} b_{\nu \lambda}\right), \\
J\left(x_{\nu}, x_{\lambda}\right)=b_{\nu \lambda}, \\
J\left(x_{\nu}, p_{\lambda}\right)=a_{\nu \lambda}, \\
J\left(p_{\nu}, x_{\lambda}\right)=-a_{\nu \lambda}, \\
J\left(p_{\nu}, p_{\lambda}\right)=b_{\nu \lambda} .
\end{gathered}
$$

If the Hamiltonian is real, as in our example, then the above equations simplify to a great extent.

The model system is a two-dimensional nonlinearly coupled anharmonic oscillator system whose classical dynamics, quantum eigenvalue spectrum, and mixed-mode behavior is studied extensively by us $[7,24,28,38]$

$$
\begin{aligned}
H= & -1 / 2\left(P_{x}^{2}+P_{y}^{2}+x^{2}+1.44 y^{2}\right)-0.05 x^{3}+0.00140625 x^{4} \\
& -0.0864 y^{3}+0.002916 y^{4}+0.1 x^{2} y^{2} .
\end{aligned}
$$

The classical trajectory calculations have shown that the critical energy for the appearance of chaos is around $E=3$ (in generalized units) and around $E=10$; the phase space is fully chaotic.

We choose the $x$ mode to be classical and the $y$ mode to be the quantal one. The initial conditions are then a single $\left(x, P_{x}\right)$ point in the phase space and a wave packet along the $y$ direction. The integration of Hamilton's equations provide the time evolution of the classical point:

$$
\begin{gathered}
\frac{\partial x}{\partial t}=P_{x} \\
-\frac{\partial P_{x}}{\partial t}=x-0.15 x^{2}+0.005625 x^{3}+0.2\left\langle y^{2}\right\rangle x^{2},
\end{gathered}
$$

where $\left\langle y^{2}\right\rangle$ denotes the expectation value over the wave packet, which has an implicit time dependence. The initial wave packet is written as a linear combination of eigenfunctions of the harmonic part of $H_{y}$ and its time evolution is governed by the appropriate Schrödinger equation,

$$
i \hbar \partial \Psi(y, t) / \partial t=\left(H_{y}+0.1 x^{2} y^{2}\right) \Psi(y, t),
$$

where $H_{y}$ contains all the terms which solely depend on $y$. At each time step, Eqs. (17) and (18) are solved keeping the external fields ( $x^{2}$ for the $y$ mode and $\left\langle y^{2}\right\rangle$ for the $x$ mode) constant and the Lyapunov exponents are computed from Eq. (14).

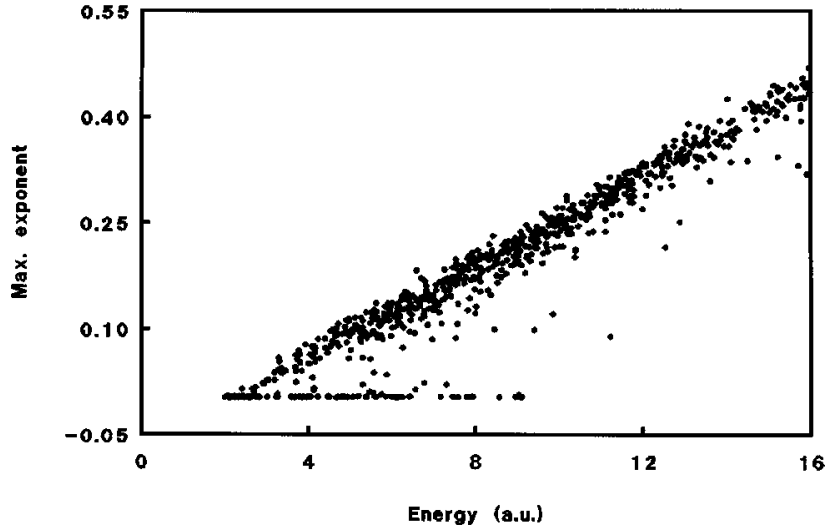

FIG. 1. Maximum Lyapunov exponents for the classical case. Exponents are in bits/a.u.

\section{RESULTS AND DISCUSSION}

Classical dynamics as a function of energy is studied by generating initial points randomly in the four-dimensional phase space. The four-point Runge-Kutta method is used for the calculation of trajectories which are integrated for 500000 steps. All Lyapunov exponents are computed with the tangent-space method with periodic orthonormalizations at every ten steps. Kolmogorov entropy, which is the sum of all positive exponents, is also stored as a function of time. Since second Lyapunov exponents are very close to zero, the qualitative behavior of the maximum exponent and Kolmogorov entropy are very similar. The plot of the maximum exponents vs energy clearly displays the onset of chaos. Around $E=2$, we start detecting positive exponents. As the energy increases, the system quickly loses its regularity and chaos settles completely around $E=8-10.0$ (Fig. 1). The small number of regular trajectories at high energy values are due to deep valleys of the potential which can trap particles. The energy along these modes cannot be transferred to the other modes within our finite integration times. The volume of the phase space for these local trajectories is almost zero.

For the mixed-mode system, the total number of Lyapunov exponents is $2 n+2$, where $n$ is the number of basis functions and at each time step one computes $2 n+2$ vectors of length $2 n+2$. The size of the resulting set of coupled equations is then $(2 n+2)(2 n+2)+(2 n+2)$ including the nonlinear equations. Consequently, the computation time increases drastically with basis size. In order to work with small basis sizes, we choose not to propagate harmonic oscillator coherent states whose expansions over harmonic oscillator eigenfunctions require rather high quantum numbers for a reasonable representation. Instead, linear combinations of low-lying eigenfunctions with real coefficients (initially at rest) are employed. We used two different initial wave packets which are

$$
\psi_{1}=\varphi_{0}+\varphi_{1}
$$

and

$$
\psi_{2}=\varphi_{2}+\varphi_{3} .
$$




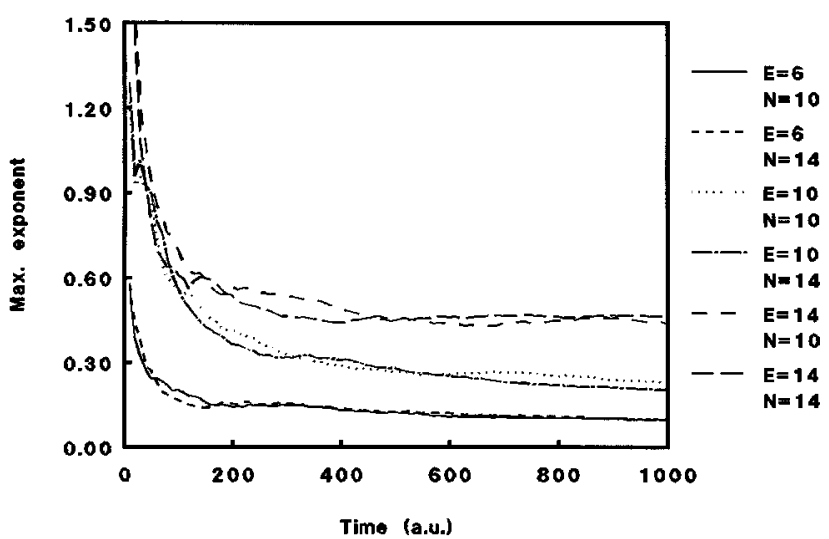

FIG. 2. Convergence of the MM exponent. Exponents are in bits/a.u.

The energies of wave packets are 1.135 and 3.267, respectively. In order to complete the picture in the mixed-mode system, three different initial conditions for the classical mode are chosen as

$$
x=0, \quad P_{x}>0, \quad x=0, \quad P_{x}<0, \quad x=1.5, \quad P_{x}>0 .
$$

Only the signs of the momenta are defined and then they are scaled in each calculation so that the total energy changes from 2.0 to 15.0 with increments of 0.5 .

In order to study the effects of the basis size, initial classical conditions, and the composition of the wave packet, we proceed to compute all Lyapunov exponents for basis sizes changing from 6 to 22 for two wave packets and three classical initial conditions. The largest set of calculations results in 46 exponents. The time steps for integrations have to be decreased by an order of magnitude with respect to the classical case in order to conserve the energy and consequently integrations are carried out for $2 \times 10^{6}$ time steps, which seems to be long enough for exponents to converge. The calculations for a single mixed-mode trajectory with 22 basis functions requires about $3 \mathrm{~h}$ of CPU time in R8000 Indigo ${ }^{2}$. In Fig. 2 the convergence of the maximum Lyapunov exponent is given for various basis sizes at different energy values. The sum of all exponents stays in the order of $10^{-13}$ and they form positive-negative pairs as they should for Hamiltonian systems [39]. The errors in pairing use in the order of $5 \%$ for small basis sizes and fall below $1 \%$ when larger basis sets are employed.

In Fig. 3(a) we display the effects of the basis size on the maximum exponent. The plotted values are averages over three classical conditions as mentioned above. At low energy, all calculations agree well and when the energy is increased, only the basis size of 6 deviates significantly from others. Therefore we conclude that a basis size of even 10 functions is sufficient to study quantum effects for this Hamiltonian, at least when the initial classical energy is high compared to the quantal one. Figure 3(b) shows a similar analysis of the effect of the initial classical condition. Here the points are averages over 10, 14, and 18 basis function results. Even the very restricted set of conditions used in these calculations indicates that the results are not very sensitive to the sampling of the classical phase space (for constant energy) or to the basis expansion provided that a suffi-
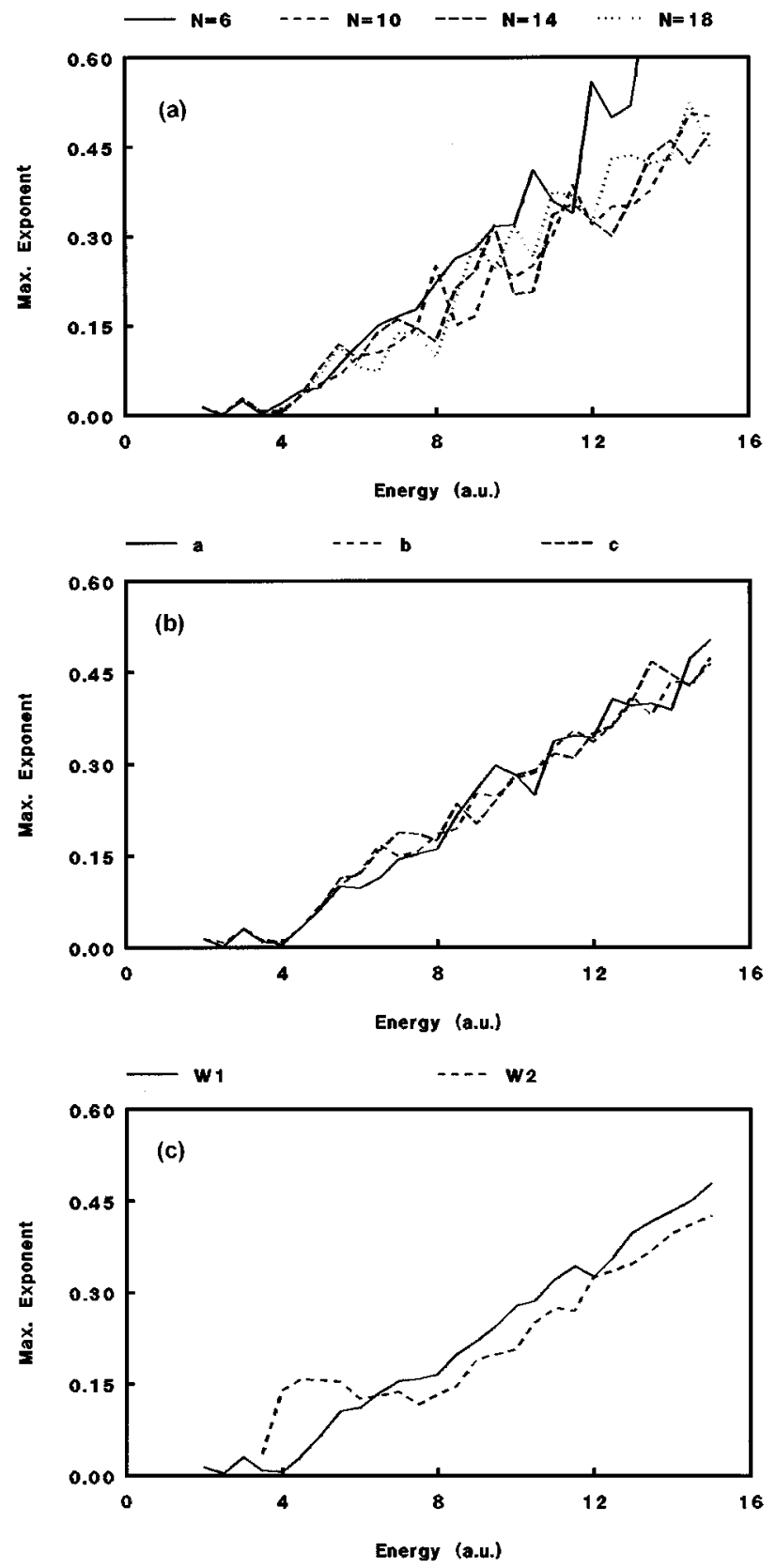

FIG. 3. (a) Dependence of Lyapunov exponents on the basis size. Exponents are in bits/a.u. (b) Dependence of Lyapunov exponents on initial classical conditions. Exponents are in bits/a.u. (c) Dependence of Lyapunov exponents on initial wave packet. Exponents are in bits/a.u.

ciently large number of functions are used. Finally, we compared the results for two different wave packets in Fig. 3(c). Again the differences due to the selection of the wave packet are very small. When one considers the fact that even the standard computations of Lyapunov exponents of classically chaotic systems give quantitatively different results for various algorithms or even under different operating systems, we believe that the above formulation of the quantal Lyapunov exponents produces reasonably precise results. We then proceed to average all results from the mixed-mode system and compare to the fully classical ones. In this averaging, two different wave packets, three different initial classical conditions, and basis sizes of 10,14 , and 18 are in- 


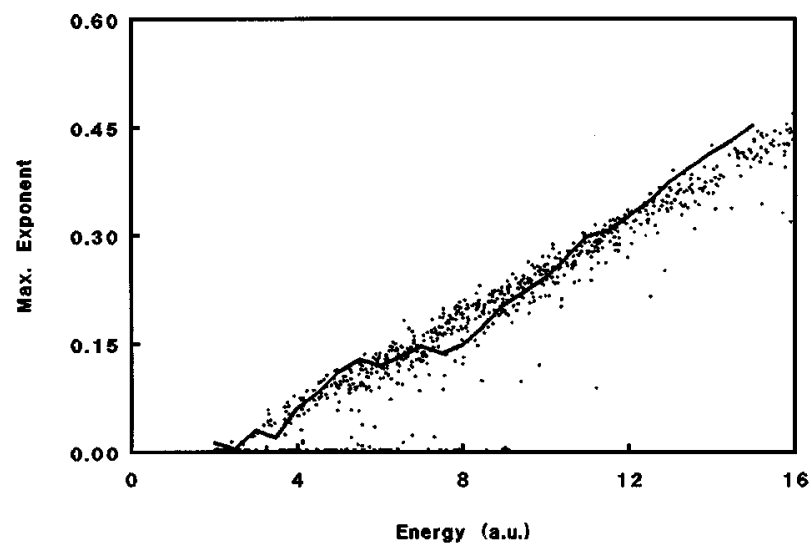

FIG. 4. Classical and MM Lyapunov exponents. Exponents are in bits/a.u.

cluded. In Fig. 4 we observe that Lyapunov exponents found for the mixed-mode approach agree extremely well with those from the fully classical ones. Similar calculations have been carried out for the simplified potential of $V(x, y)=0.5\left(x^{2}+y^{2}+x^{2} y^{2}\right)$ which has a very high symmetry. The agreement between the mixed-mode and classical Lyapunov exponents again is very good.

A systematic comparison between the mixed-mode and classical trajectories will be presented elsewhere. Here the basic difficulty lies in the phase space representation of the wave packet. The selection of a set of initial classical points for a given wave packet is not unique. The Wigner transformation [40] is asymptotically correct; however, it cannot be strictly used as a probability distribution since it is not positive in all regions. On the other hand, the Husimi distribution [41,42], which is a Gaussian smoothed Wigner transformation, can be used for such correspondence between a wave packet and a bundle of trajectories, but it lacks a rigorous proof. Here we would like to compare only three trajectories at different energy values. In Fig. 5, these trajectories are given on the $y-p_{y}$ plane, which is the quantized coordinate in the mixed-mode representation. The lighter dots are for the classical dynamics and the darker points correspond to the expectation values of the wave packet. At all energy values, different portions of the phase space are visited. Mixed-mode trajectories are mostly located around the origin due to the averaging involved in the expectation values. At $E=2.0$, both classical and MM results are regular even though they span different parts of the phase space. The classical particle avoids the origin since the energy stored in the $y$ mode is always large. In contrast the wave packet is making small amplitude motion around the origin. As the energy is increased, the classical particle starts to visit all cells in the phase space and the MM trajectory does not follow any periodic orbits.

Previously we have shown that a partial quantization smoothes out the chaotic details of the motion of the classical particle [24]. Applying the same methodology, now we observe that a quantum system under the effect of a classical field behaves just like a classical particle under similar conditions, that it displays "chaos." Before a claim for the "quantum chaos" can be made, one has to analyze this rather unexpected behavior. Two fundamentally different approaches of classical and quantum mechanics are connected
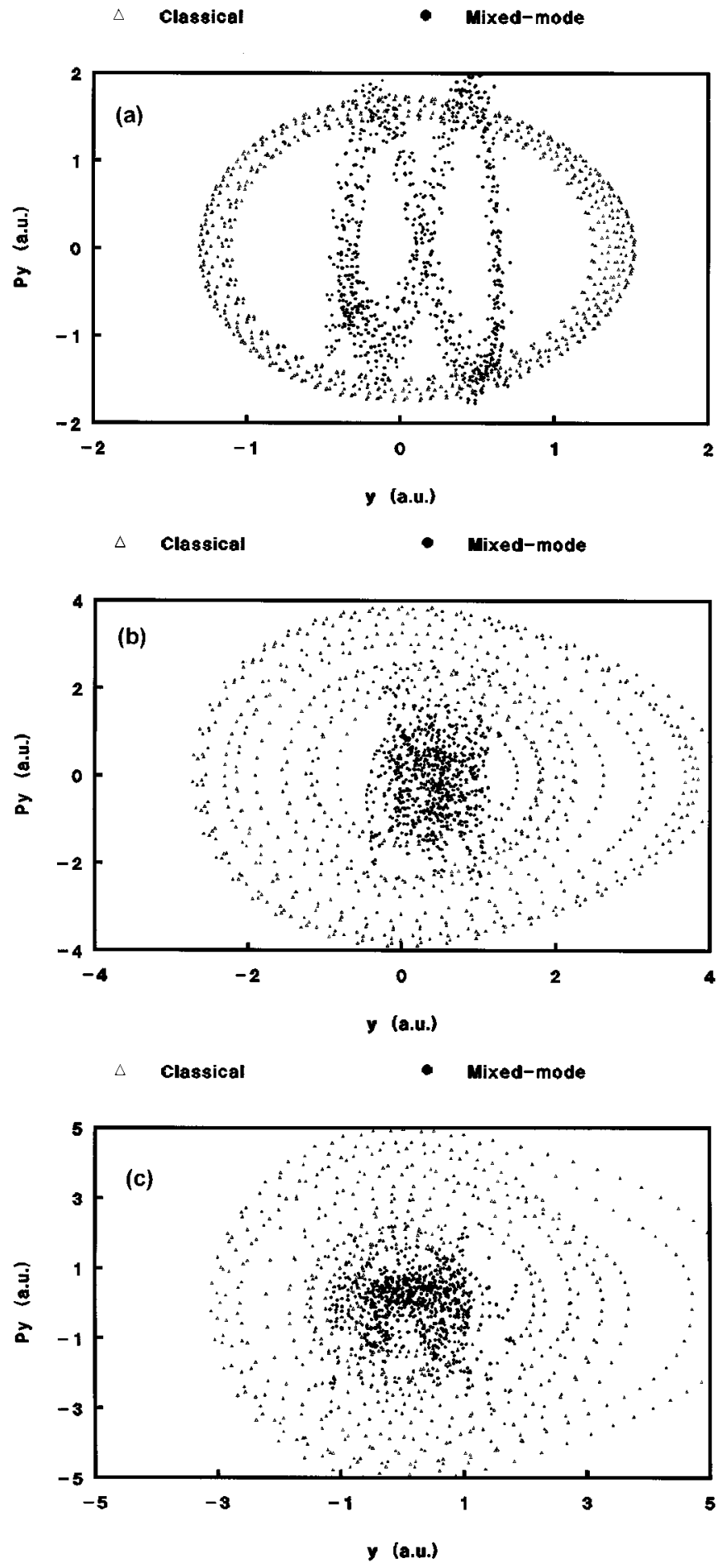

FIG. 5. (a) Classical and MM trajectories at $y$ - $p_{y}$ plane for $E=2.0$ a.u. Exponents are in bits/a.u. (b) Classical and MM trajectories at $y-p_{y}$ plane for $E=8.0$ a.u. Exponents are in bits.a.u. (c) Classical and MM trajectories at $y-p_{y}$ plane for $E=15.0$ a.u. Exponents are in bits/a.u.

via Ehrenfest couplings which are highly unsymmetric. When a quantum particle moves under a classical field, the chaotic details of the classical motion are carried over exactly into the Schrödinger equation; that is, there is no averaging process for the field. On the other hand, in classical dynamics, quantum fields appear only as expectation values which will wash out all the details, destroying chaos. There- 
fore, the channel which allows the energy transfer between two different worlds actually decides on the nature of the dynamics by either carrying over all the minute details or by selecting basics.

\section{ACKNOWLEDGMENT}

This work is partially supported by a grant from Volkswagen Stiftung, No. 1 69/797.
[1] M. C. Gutzwiller, Chaos in Classical and Quantum Mechanics (Springer-Verlag, Berlin, 1990).

[2] F. Haake, Quantum Signatures of Chaos (Springer-Verlag, Berlin, 1991).

[3] Quantum Chaos, edited by H. A. Cardeira, R. Ramaswamy, M. C. Gutzwiller, and G. Casati (World Scientific, Singapore, 1991).

[4] G. Casati and B. Chirikov, Physica D 86, 220 (1995).

[5] A. Wolf, J. B. Swift, H. L. Swinney, and J. A. Vastano, Physica D 16, 288 (1985).

[6] J.-P. Eckmann, S. O. Kamphorst, D. Ruelle, and S. Ciliberto, Phys. Rev. A 34, 4971 (1986).

[7] E. Yurtsever and J. Brickmann, Ber. Bunsenges. Phys. Chem. 94, 804 (1990).

[8] E. J. Heller, in Chaos and Quantum Physics, edited by A. Voros, M. Gianonni, and O. Bohigas (North Holland, Amsterdam, 1990).

[9] D. Delande, Comments At. Mol. Phys. 25, 281 (1991).

[10] R. E. Prange, in Quantum Chaos (Ref. [3]), Chap. 1.

[11] M. Shapiro, J. Ronkin, and P. Brumer, Ber. Bunsenges. Phys. Chem. 92, 212 (1988).

[12] T. A. Brody, J. Flores, J. B. French, A. P. Mello, A. Pandey, and S. S. M. Wong, Rev. Mod. Phys. 53, 385 (1982).

[13] T. Zimmermann, L. S. Cederbaum, H-D. Meyer, and H. Köppel, J. Phys. Chem. 91, 4447 (1987).

[14] J. Karwowski, M. Bancewicz, O. N. Ventura, and G. H. F. Diercksen, J. Phys. A 26, 5581 (1993).

[15] B. V. Chirikov, in Quantum Chaos (Ref. [3]), p. 57.

[16] M. V. Berry and M. Robnik, J. Phys. A 17, 2413 (1984).

[17] J. Brickmann and R. D. Levine, Chem. Phys. Lett. 120, 252 (1983).

[18] J. Brickmann, Y. M. Engel, and R. D. Levine, Chem. Phys. Lett. 137, 441 (1987).

[19] D. W. Noid, M. L. Koszykowski, and R. A. Marcus, Annu. Rev. Phys. Chem. 32, 267 (1981).
[20] E. Yurtsever and J. Brickmann, Phys. Rev. A 38, 1027 (1988).

[21] M. Toda and K. Ikeda, Phys. Lett. A 124, 165 (1987).

[22] U. Schwengelbeck and F. H. M. Faisal, Phys. Lett. A 199, 281 (1995).

[23] T. Uzer, Phys. Rep. 199, 73 (1991).

[24] E. Yurtsever, Phys. Rev. E 50, 3422 (1994).

[25] D. Farrely and A. D. Smith, J. Phys. Chem. 90, 1599 (1986).

[26] R. Alimi, R. B. Gerber, A. D. Hammerich, R. Kosloff, and M. A. Ratner, J. Chem. Phys. 93, 6484 (1990).

[27] D. Farrely and M. D. Emmel, Chem. Phys. Lett. 217, 520 (1993).

[28] E. Yurtsever and J. Brickmann, Ber. Bunsenges. Phys. Chem. 96, 145 (1992).

[29] E. Yurtsever and T. Uzer, Ber. Bunsenges. Phys. Chem. 96, 906 (1992).

[30] H-D. Meyer, U. Manthe, and L. S. Cederbaum, Chem. Phys. Lett. 165, 73 (1990).

[31] R. B. Gerber, V. Buch, and M. A. Ratner, J. Chem. Phys. 77, 3022 (1982).

[32] R. Alimi, A. Garcia-Vela, and R. B. Gerber, J. Chem. Phys. 96, 2034 (1992).

[33] E. Yurtsever and J. Brickmann, in Frontiers of Chemical Dynamics, edited by E. Yurtsever (Kluwer, Dordrecht, 1995), p. 393.

[34] Y. Guan, J. T. Muckerman, and T. Uzer, J. Chem. Phys. 93, 4400 (1990).

[35] E. Yurtsever and J. Brickmann, Ber. Bunsenges. Phys. Chem. 98, 554 (1994).

[36] T. Gunkel, H-J. Baer, M. Engel, E. Yurtsever, and J. Brickmann, Ber. Bunsenges. Phys. Chem. 98, 1552 (1994).

[37] R. J. Hinde, R. S. Berry, and D. J. Wales, J. Chem. Phys. 96, 1376 (1992).

[38] E. Yurtsever and J. Brickmann, Phys. Rev. A 41, 6688 (1990).

[39] H-D. Meyer, J. Chem. Phys. 84, 3147 (1986).

[40] E. P. Wigner, Phys. Rev. 40, 749 (1932).

[41] K. Husimi, Proc. Phys. Math. Soc. Jpn. 22, 264 (1940).

[42] P. A. Jalil and E. Yurtsever, Turkish J. Phys. 18, 1095 (1994). 